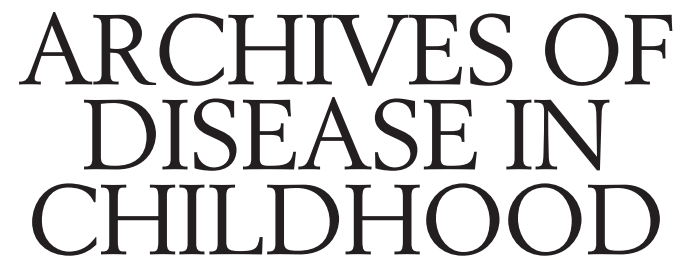

The Fournal of the Royal College of Paediatrics and Child Health

\title{
Annotations
}

\section{Psychological aspects of hemiplegia}

Do paediatricians think of childhood hemiplegia simply as a mild physical disability? Rightly or wrongly, parents often get that impression. This is unfortunate as children and families are easier to help when they know that their difficulties are not being underestimated. The disability does not seem mild to hemiplegic children who are always last on sports day, or self conscious about their body, or unable to carry out all manner of mundane two handed activities. Furthermore, hemiplegia is often more than a physical problem, being accompanied by a variety of 'invisible' psychological disabilities. Particularly in the school years, many parents and children are more concerned about the educational, behavioural, emotional, and social accompaniments of hemiplegia than about the physical disability itself. Some of these psychological accompaniments are understandable responses to disability; others are direct consequences of the brain damage itself; and many reflect the complex interplay of these reactive and organic pathways.

\section{Cognitive difficulties}

Though approximately two thirds of children with hemiplegia are of normal intelligence, the mean IQ of the group is shifted downwards, most markedly for those with the greatest neurological involvement. ${ }^{12}$ Hemiplegias acquired between 1 and 60 months of age may be particularly liable to reduce overall intelligence, perhaps because this is a peak period for synaptic creation and remodelling. ${ }^{2}$

Many IQ tests distinguish between verbal IQ, based on tests of verbal reasoning, and performance IQ, based on puzzles and other non-verbal reasoning tests. The ability of children with hemiplegia is usually best judged from their verbal IQ. Performance IQ is often 10 or more points lower, partly because hemiplegic children are disadvantaged by tests that require two handed skill, and partly because the developing brain's response to damagewhether to the left or the right hemisphere-seems to be to preserve language skills at some cost to visuospatial skills. ${ }^{23}$ This is fortunate as most scholastic skills depend more on verbal than visuospatial abilities - though the specific visuospatial difficulties do sometimes cause problems in areas such as map reading, geometry, or electronics.

Even when intelligence has been taken into account, children with hemiplegia are more liable to specific learning difficulties affecting reading, spelling, or arithmetic. ${ }^{4}$
The recognition and treatment of specific learning difficulties is important for any child, but is particularly important for a child who will eventually need to seek a non-manual job because physical disabilities preclude manual employment.

\section{Emotional and behavioural problems}

Roughly half of all children with hemiplegia have psychiatric disorders - that is, problems with behaviour, emotions, or relationships that interfere markedly with the child's everyday life, cause substantial distress for the child, or result in considerable disruption for others. ${ }^{5}$ The commonest presenting features are irritability, anxiety, and hyperactivity/inattention - all of which seem more related to the underlying brain damage than to the child's environment. Irritability usually goes along with marked temper tantrums and lack of compliance with adult requests. Although these children may be aggressive when provoked, they rarely show a 'delinquent' pattern of deliberate aggression and antisocial behaviour. Anxiety may show itself in generalised worrying, excessive fear of separations, marked shyness, or specific phobias. Severe and persistent misery is relatively uncommon and often seems to be related to school stresses: placement in the wrong sort of school, unrecognised special needs, or victimisation.

In a society where children's misbehaviour is normally attributed to parental mishandling, it is not surprising that parents often blame themselves or feel blamed by others if their hemiplegic children develop emotional or behavioural problems. In most cases, however, family factors seem less important than biological and school factors. When family stress is associated with psychological problems in the child, it is often the child's problems that are driving the family stress rather than vice versa. Even when the child's behaviour is worse at home than at school, this does not necessarily point to family factors. Some children are well behaved at school despite teasing or insufficient academic support, but then vent their pent-up rage and distress on family members after school-improvement during school holidays is a helpful pointer.

Parents and professionals often assume that the psychological consequences of static brain disorders are particularly resistant to any sort of treatment. The underlying logic seems to be that as the brain damage is permanent, and as the psychological consequences stem from the brain 
damage, those consequences will also be permanent. This is faulty logic - on the same basis no one would treat the epilepsy of children with cerebral palsy. Nevertheless, gloomy prognoses can be self fulfilling if they stand in the way of initiating treatment. In many cases, children with hemiplegia and psychiatric problems are more responsive to treatment than children whose psychiatric problems stem from chronic psychosocial adversity. With highly motivated parents, a relatively small amount of informed advice can go a long way. In addition, specific disorders seem likely to respond to the same sorts of specific treatments - such as medication, behavioural therapy, and family therapy-used for other children with comparable psychiatric presentations.

\section{Problems with peer relationships}

Even though most children with hemiplegia are integrated in mainstream schools, problems getting on with other children are common. While most hemiplegic children do have some playmates, they have fewer close reciprocal friendships than most of their classmates, and they are substantially more likely to be teased or bullied. ${ }^{6}$ Prejudice may play a part, but there is increasing evidence for constitutional impairments in social understanding. There is growing interest in children's abilities to understand other people's perspectives, beliefs and intentions; the development of these abilities appears to be delayed in children with hemiplegia, contributing to their emotional and social immaturity.

\section{Practical help}

As a child health professional, what can you do to prevent and treat the psychological complications of your patients with hemiplegia? First, put the issue on the agenda: show through your attitude and questions that you are concerned about psychological as well as physical development. Parents are often greatly reassured to find that their child's problems are common consequences of hemiplegia; the energy previously locked up in self blame can then be diverted into more profitable channels. By telling the family about Hemi-Help, the nationwide parents' support group, you can further reduce the family's sense of isolation and powerlessness by providing them with access to regular newsletters, meetings, and advice (inquiries and help line: 0181-672-3179). Ensuring appropriate educational provision may also depend, at least in part, on health service input. Adequate neuropsychological assessment helps delineate the child's needs and the best ways of meeting those needs; in some areas this assessment is most appropriately carried out by clinical rather than educational psychologists. Reassessment is advisable if a child is running into psychological problems, particularly if the child is becoming hopelessly demoralised by lack of success in all areas of school life. Finally, if the child's psychological problems seem too complicated to assess or treat locally, refer on.

\section{ROBERT GOODMAN}

Department of Child and Adolescent Psychiatry,

Institute of Psychiatry,

De Crespigny Park,

London SE5 $8 A F$

1 Goodman R, Yude C. Do incomplete ascertainment and recruitment matter? Dev Med Child Neurol 1996;38:156-65.

2 Goodman R, Yude C. IQ and its predictors in childhood hemiplegia. Dev Med Child Neurol 1996;38:881-90.

3 Vargha-Khadem F, Isaacs E, Muter V. A review of cognitive outcome after unilateral lesions sustained during childhood. 7 Child Neurol 1994;9:2S672S73.

4 Muter V. Phonology and learning to read in normal and hemiplegic children. (PhD thesis.) London: University of London, 1994.

5 Goodman R, Graham P. Psychiatric problems in children with hemiplegia: cross sectional epidemiological survey. BMF 1996;312:1065-9.

6 Yude C. Peer problems of children with hemiplegia in mainstream schools. (PhD thesis.) London: University of London, 1996

7 Balleny H. Are the concepts of 'theory of mind' and 'executive function' useful in understanding social impairment in children with hemiplegic cerebral palsy. (Clin Psy D thesis.) Norwich: University of East Anglia, 1996.

\section{Preventative strategies on meningococcal disease}

The dramatic occurrence of meningococcal disease challenges doctors and attracts intense public interest. It is treatable and preventable, so every death raises the question: Could this have been avoided? Last winter's experience of clusters in schools was the latest round in a struggle to apply rational and effective policies to control disease and panic. A strategist planning to prevent the occurrence and damage of meningococcal disease has various forces to deploy: epidemiology, the recognition of people who are susceptible to infection, vaccines, antibiotics for prophylaxis and treatment, public awareness, and education of doctors and parents. The epidemiology of meningococcal infection is fundamental to any preventive strategy. The incidence in the UK is $2.5-3$ cases/100 000/ year, 1200 to 2000 cases, with exact numbers depending on case definitions and the year in question. ${ }^{12}$ The age specific annual incidence peaks at 6 months of age (50-60/ 100000 ), falls to $2 / 100000$ at age 10 years, rises slightly to $5 / 100000$ in teenagers, and then falls to $1 / 100000$ in adults. $^{3}$

Meningococcal disease illustrates the two purposes of notification: to start public health action and to have statistics on incidence. The consultant in communicable disease control must be notified, to ensure that prophylaxis and advice is given to close contacts and that local general practitioners, schools, and nurseries are informed. A prevention strategy depends on a reliable count of cases. Notifications are an enduring source of statistics, but there has been under-reporting in the past. In the last three years there have been fewer laboratory isolates than notifications, which produced problems in the autumn of 1995 when the public were aware of clusters and an increase in notifications, which was not substantiated by laboratory reports. ${ }^{4}$ The only reliable way to enhance surveillance is to combine sources of data. The laboratory reporting system is accurate for diagnosis, but is an underestimate of incidence since the widespread use of preadmission penicillin, which reduces the proportion of laboratory confirmed cases. Caution in performing lumbar punctures on children may also reduce the numbers confirmed by microbiology. The gap can be reduced by serology, culturing throat swabs from patients on admission and by antigen detection (for example the polymerase chain reaction). ${ }^{5}$ Microscopy of fluid from purpuric lesions is a technique that is sometimes forgotten.

Meningococci are spread by respiratory droplets from the nasopharynx. Disease, if it is going to occur, is within two to 10 days of contact. If illness does not occur, the exposed person who is colonised acquires immunity ${ }^{6}$ but becomes a possible source of infection to others who are exposed to his or her respiratory droplets. 